\title{
Ray Tracing Simulations of ECR Heating and ECE Diagnostic at W7-X Stellarator
}

\author{
Nikolai B. MARUSHCHENKO, Volker ERCKMANN, Hans J. HARTFUSS, Mattias HIRSCH, \\ Heinrich P. LAQUA, Henning MAASSBERG and Yuri TURKIN \\ Max-Planck-Institut für Plasmaphysik, EURATOM-Association, Wendelsteinstr. 1, D-17491 Greifswald, Germany
}

(Received 4 December 2006 / Accepted 9 March 2007)

This report shows the progress achieved in simulations of thermal and non-thermal effects which may appear for high power ECRH in the W7-X stellarator (under construction in Greifswald, Germany). Simulations are carried out with the new ray tracing code TRAVIS which was developed for electron cyclotron studies in arbitrary 3D magnetic configurations, with emphasis on heating, current drive and ECE diagnostic.

(c) 2007 The Japan Society of Plasma Science and Nuclear Fusion Research

Keywords: ECR heating, current drive, ECE diagnostic, ray tracing

DOI: $10.1585 /$ pfr.2.S1129

\section{Introduction}

The W7-X stellarator is a large-scale device (average major radius $R_{0} \simeq 5.5 \mathrm{~m}$, and plasma radius $a \simeq 0.55 \mathrm{~m}$ ), equipped with superconducting coils, with a low-shear configuration of the Helias (Helical Advanced Stellarator) type [1] with five field periods. The W7-X configuration has a strong variation of the plasma shape as well as a strongly varying magnetic field. While the "bean-shaped" plane corresponds to the maximum $B$ region, the "triangular" plane has the minimum of $B$. For the "standard" configuration with $B_{\max } / B_{\min }=1.09$, the trapped particles fraction on axis is $f_{\mathrm{tr}} \simeq 0.3$.

The W7-X will demonstrate the inherent steady state capability of stellarators at reactor relevant parameters. The total power of the ECRH system will reach $10 \mathrm{MW}$ for different heating scenarios. The expected range of electron temperatures is quite high (for regimes with low and moderate densities up to $10 \mathrm{keV}$ and more, as predicted by transport modelling [2]), and simulations of ECR heating, as well ECE diagnostic, require to take into account the appearance of non-thermal effects. Below, we describe the structure of the new ray tracing code TRAVIS and show the results of such simulations for quite different problems, ECRH and ECE diagnostic.

\section{Ray Tracing Code TRAVIS}

The new ray tracing code TRAVIS (TRAcing VISualised) was developed for electron cyclotron studies in arbitrary 3D magnetic configurations, with emphasis on heating, current drive (CD) and ECE diagnostic. The magnetic configuration provided by the $3 \mathrm{D}$ equilibrium code VMEC is converted to Boozer co-ordinates and rapidly interpolated by a specially developed highly optimized package. The code is used through an especially designed

author's e-mail: nikolai.marushchenko@ipp.mpg.de graphical user interface, which allows the preparation of input parameters and viewing results in convenient $(2 \mathrm{D}$ and 3D) form. The aim of this interface is to make the code suitable for any interested user.

The ray tracing equations are the standard Hamiltonian ones (see, e.g. [3]),

$$
\frac{d \mathbf{r}}{d s}=\frac{\partial \mathcal{H}}{\partial \mathbf{k}} /\left|\frac{\partial \mathcal{H}}{\partial \mathbf{k}}\right|, \quad \frac{d \mathbf{k}}{d s}=-\frac{\partial \mathcal{H}}{\partial \mathbf{r}} /\left|\frac{\partial \mathcal{H}}{\partial \mathbf{k}}\right|,
$$

where $\mathbf{r}$ is the radius-vector, $\mathbf{k}$ is the wave-vector, and $s$ is the path along the ray. For the Hamiltonian, $\mathcal{H}$, the most general form suggested by Tokman and Westerhof $[4,5]$ is adopted. Strictly speaking, the code includes both models Eq.(2), of Westerhof, $\mathcal{H}=\mathcal{H}^{W}$, and of Tokman, $\mathcal{H}=\mathcal{H}^{T}$,

$$
\mathcal{H}=\left\{\begin{array}{l}
\mathcal{H}^{W}=N^{2}-N_{\|}^{2}-\left(\mathfrak{R} N_{\perp}\right)^{2}, \\
\mathcal{H}^{T}=\mathfrak{R}\left(D_{i j}^{H} e_{i}^{*} e_{j}\right),
\end{array}\right.
$$

which, being based on the same physics, produce almost identical results (here, $N_{\perp}$ is the root of the complete dispersion relation, $\operatorname{det} D_{i j} \equiv\left\|N^{2} \delta_{i j}-N_{i} N_{j}-\epsilon_{i j}\right\|=0$, $\epsilon_{i j}=\epsilon_{i j}^{H}+i \epsilon_{i j}^{a H}$ is the weakly relativistic dielectric tensor, expanded to the Hermitian, $\epsilon_{i j}^{H}$, and anti-Hermitian, $\epsilon_{i j}^{a H}$, parts, and $e_{i}$ is the $i$-th component of the dimensionless polarization vector, calculated from $\epsilon_{i j}$ ). Generally, the "cold", "warm" non-relativistic or weakly relativistic dielectric tensor can be used in the Hamiltonian. With the weakly relativistic dielectric tensor, the model of tracing includes those kinetic effects which become a significant in the vicinity of the EC resonance, leading to "anomalous" dispersion effects and possible bending of the rays $[4,5]$. For example, in contrast to the "cold" approach (which should be sufficient for many cases), the weakly relativistic model can give a quite different result in the case of quasivertical launch with the ray trajectories almost tangential to the resonance line. 
In the code, the absorption $\alpha_{\omega}$ and the emissivity $\eta_{\omega}$ are calculated for an arbitrary electron distribution function, $f_{e}$, from the anti-Hermitian part of the fully relativistic dielectric tensor, $\epsilon_{i j}^{a H}\left(f_{e}\right)$, and from the micro-current correlation tensor, $G_{i j}\left(f_{e}\right)$, respectively (see, e.g. [6]). The electron distribution function $f_{e}$ used for calculations can be Maxwellian, bi-Maxwellian or arbitrary (numerically given), thus allowing future coupling with a Fokker-Planck solver. During the integration along the resonance curve, apart of calculation the energy range of electrons responsible for absorption, by analyzing the value of the magnetic moment, the wave-particle interaction is calculated separately for passing and trapped electrons. The $\mathrm{CD}$ efficiency is calculated by adjoint approach with momentum conservation $[7,8]$ (the simplified "high-speed limit" model [9] without momentum conservation also is included).

For the simulation of the ECE spectrum, special options are included to estimate the theoretical limit of spatial resolution (spatial width of the emission line) and to identify the energy range of electrons dominating in the emission (this might be used to diagnose fast electrons). The radiative temperature is calculated as solution of the radiative transport equation (multiple reflection is omitted),

$$
T_{\omega}^{\text {ece }}=\left\langle C g_{\omega} \sum_{\text {rays }} w_{\text {ray }} \int_{s_{0}}^{s_{1}} \mathrm{~d} s^{\prime} \eta_{\omega}\left(s^{\prime}\right) \mathrm{e}^{-\tau_{\omega}\left(s_{1}\right)+\tau_{\omega}\left(s^{\prime}\right)}\right\rangle_{\Delta \omega},
$$

where $C=8 \pi^{3} c^{2} / \omega^{2}, \quad s$ is the path along the ray, and $\tau_{\omega}(s)=\int_{s_{0}}^{s} \mathrm{~d} s^{\prime} \alpha_{\omega}\left(s^{\prime}\right)$ is the optical depth. The antenna beam with Gaussian pattern is discretized by the number of rays, giving the ray weight factor as $w_{\text {ray }} \propto \mathrm{e}^{-\rho_{\text {ray }}^{2} / 2 b^{2}}$ ( $\rho_{\text {ray }}$ is the ray radius, and $b$ is the beam cross-section size). For each frequency, $\omega$, the results are averaged over the $\omega \pm \Delta \omega / 2$ range with the correspondent frequency band function, $g_{\omega}$, which is the radiometer characteristic.

The code is benchmarked against the "old" W7-AS code [8] and the WR_RTC [10]. Additionally, the code was successfully tested on the ITER reference "Scenario2" against several other predictions (TORRAY, GENRAY, CQL3D, etc.) The code is now routinely exploited in modeling heating at various harmonics of the ordinary and extraordinary mode $(\mathrm{O} 1, \mathrm{O} 2, \mathrm{X} 2$ and $\mathrm{X} 3)$ in different magnetic configurations. The code is also supporting the design of ECRH launcher components for W7-X.

\section{O2-Mode, High-Field-Side Launch}

Two ports close to the "triangular" plane $\left(\phi=36^{\circ}\right)$ are available at W7-X for physics investigations with highfield-side (HFS) launch of ECRH with a maximum power of $2 \mathrm{MW}$. The magnetic field shows a weak inverse gradient in this plane.

The simulation is performed for $B_{0}=2.7 \mathrm{~T}$ (the axial value at $\phi=0), n_{e}=1.5 \times 10^{20} \mathrm{~m}^{-3}$, and $T_{e}=3$ $\mathrm{keV}$. The Gaussian beam with initial width of $10 \mathrm{~cm}$ is discretized by 80 rays. Because of high density, refraction

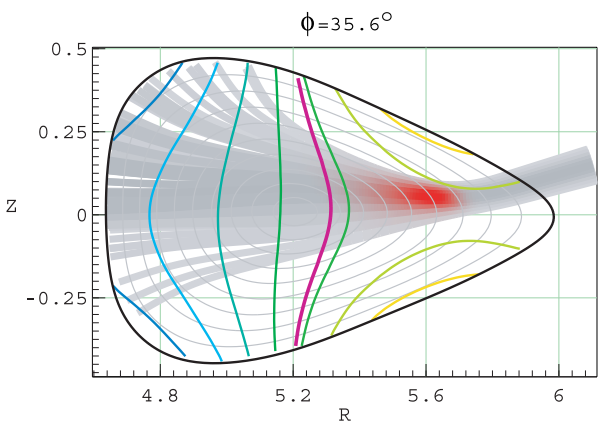

Fig. 1 Ray trajectories for O2-mode for HFS launch in "triangular plane". The resonance line, $B=2.5 \mathrm{~T}$, is also shown (thick violet line).
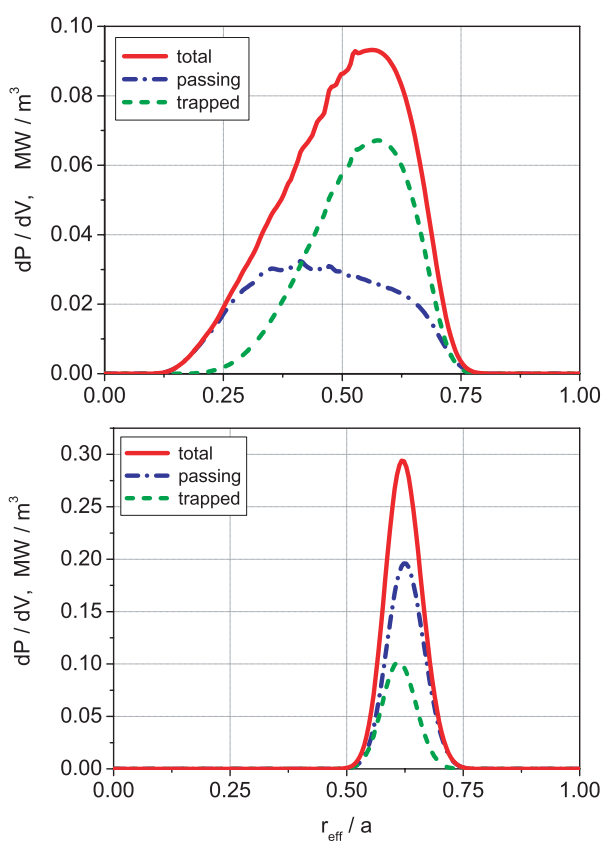

Fig. 2 HFS launch in "triangular plane": deposition profiles for both trapped (green) and passing (blue) electrons, and the total one (red line). Top - O2-mode, bottom - X2-mode.

is significant and does not allow the focusing of the beam (Fig. 1). Since the plasma is "optically gray" for O2-mode, the deposition profile, $p\left(r_{\text {eff }}\right)$, is quite broad (Fig. 2, top), and the power shine-through is about $13 \%$. The most important result (and even somewhat surprising for O-mode) is that about $60 \%$ of power is absorbed by trapped electrons. Furthemore, the shapes of the deposition profiles for trapped and passing electrons ( $p_{\mathrm{tr}}$ and $p_{\mathrm{pa}}$, respectively, with $\left.p=p_{\text {tr }}+p_{\mathrm{pa}}\right)$ are different.

The damping along the ray becomes significant from $r_{\text {eff }} / a \leq 0.7$ with electron velocities $v / v_{\text {th }} \simeq 3$, where the absorption by trapped electrons is dominant. In the region $r_{\text {eff }} / a<0.4$, where the velocity range is decreased up to $v / v_{\text {th }} \simeq 1.5$, mainly passing electrons are responsible for absorption. The optical depth is still not so high there, $\tau \simeq$ $1-1.5$ (plasma is gray), and the rays contain quite enough 
power to heat also passing electrons.

For comparison, in Fig. 2 (bottom) also the deposition profiles for X2-mode for equivalent conditions (only the density reduced to $n_{e}=0.5 \times 10^{20} \mathrm{~m}^{-3}$ ) are shown. About $70 \%$ of power is absorbed by passing electrons, and the profiles for both trapped and passing electrons have almost the same shape. Note, that this $\mathrm{O} 2$ feature is specific for W7-X and for the HFS launch scenario. It demonstrates a flexibility of W7-X, where the fraction of trapped particles in the "triangular plane" can be varied in configuration scans. This adds to the flexibility of the launcher.

\section{ECE Diagnostic, LFS vs HFS Ob- servations}

The standard scheme of low-field-side (LFS) ECE measurements at the X2-mode is based on i) a good (spatial) localization of the "emission line", which means a high optical thickness of the plasma for the observed radiation and sufficiently high $\nabla B$ along the sightline, and ii) a Maxwellian electron distribution function. While for the appropriate frequency range the first condition is well satisfied (the ECE diagnostic system is planned to be installed near the "bean-shaped" plane, where $\nabla B$ is largest), the second one can be violated, especially for low density ECR heated plasmas. In the standard LFS observation scheme, the main contribution in the emission is produced by bulk electrons with energies of not more than roughly $2 T_{e}$, i.e. the measured ECE spectrum is close to the thermal one.

The detection of non-thermal effects requires a special technique. A HFS observation along the same sightline as the LFS one $[11,12]$ appears to be very promising: despite of the moderate spatial resolution of a HFS ECE diagnostic, the existence of supra-thermal electrons can be identified by comparison of both LFS and HFS ECE spectra. Abilities of a HFS diagnostic as a tool to indicate the emission of supra-thermal electrons in tokamaks have been examined both theoretically and experimentally (see, e.g. $[13,14])$, where the high sensibility of the non-thermal ECE is confirmed. Nevertheless, the interpretation of the HFS measured ECE spectra is not trivial and requires special attention.

The careful "mapping" the ECE spectrum onto the proper magnetic surfaces is also requested. Due to the relativistic (and Doppler) broadening, a non-locality of the emission line arises, when the gradients of density and temperature become important. Instead of the "cold" resonance position, $\mathbf{X}_{\mathrm{cy}}$ for a given frequency, one needs to find the "weighted" center of the emission line, $\mathbf{X}_{\text {ece }}$, and to estimate its spatial width, $\Delta X_{\text {ece }}$, (i.e. the spatial resolution). For these calculations, a similar algorithm as in [15] is used: the point at the sightline, where half of the integral emission intensity is reached, is called the center of the (asymmetric) emission line. Including in the integral emission intensity only $90 \%$ of the total value, the respective width of the emission line is estimated by cutting off the
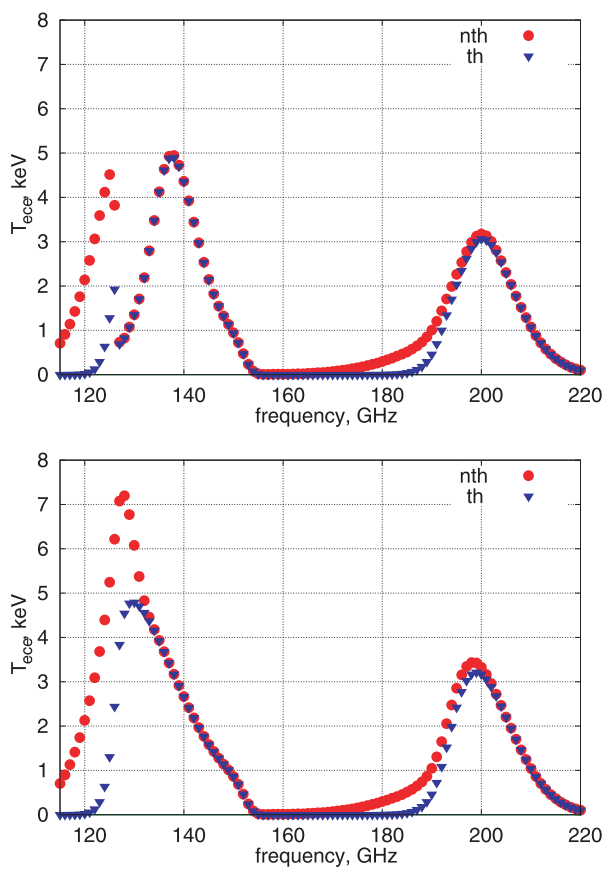

Fig. 3 ECE spectra for LFS (top) and HFS (bottom) observations for both Maxwellian (blue triangles) and biMaxwellian (red points) distribution functions.

rest of its wings. As the final step, the "weighted" center of the emission line, $\mathbf{X}_{\text {ece }}$, together with its wings are mapped onto the magnetic coordinates, producing $r_{\text {eff }}\left(\mathbf{X}_{\text {ece }}\right)$ and the appropriate "error bars". The same technique is applied for estimation the energy range of absorbing electrons.

For (preliminary) estimations of the non-thermal contributions in the ECE spectrum, it is sufficient to use a simple bi-Maxwellian model. The electron distribution function is represented as $f_{e}=(1-\delta) f_{M 0}+\delta f_{M 1}$, with $f_{M 0}$ and $f_{M 1}$ being Maxwellian distribution functions, the main one and the supra-thermal one, respectively. For the highly localized ECRH deposition profile, it is also assumed, that the supra-thermal fraction exists only near the axis, $r_{\text {eff }} \lesssim 5 \mathrm{~cm}$. In this paper, the supra-thermal fraction is assumed as $\delta=0.05$ and $T_{e 1}(0) / T_{e 0}(0)=3$, which corresponds to $15 \%$ of the energy contained in supra-thermal electrons in the plasma center. This model is more or less adequate to the expected quasi-linear flattening of the electron distribution function, producing a non-negligible supra-thermal fraction in the most interesting range, $v / v_{\text {th }} \sim 1$ 1.5-3. Simulations are performed for $n_{e}(0)=2 \times 10^{19} \mathrm{~m}^{-3}$ with an almost flat profile near the axis (within the heated region), and for a peaked $T_{e}$ profile with $T_{e}(0)=5 \mathrm{keV}$.

For $B_{0}=2.5 \mathrm{~T}$, the magnetic field varies along the ECE observation chord between $2.25 \mathrm{~T}$ and $2.82 \mathrm{~T}$, and the resonance frequencies for the neighboring harmonics (2nd and 3rd) have no overlap (from $126 \mathrm{GHz}$ to $158 \mathrm{GHz}$ and from $189 \mathrm{GHz}$ to $237 \mathrm{GHz}$, respectively). The frequency range from $115 \mathrm{GHz}$ to $220 \mathrm{GHz}$, used for the simulations, 
covers both the 2 nd and the 3 rd harmonics ranges (below we analyse only the 2 nd harmonic range). The lower frequency limit (2nd harmonic) is chosen to cover the downshifted emission from the supra-thermal electrons for both HFS and LFS cases. The beam collected by the ECE antenna is modeled by a bundle of rays, the intensity of the beam is distributed among the rays according to a Gaussian antenna pattern. Following Eq.(3), for each frequency, $\omega=2 \pi f$, the results are averaged over the $f \pm \Delta f / 2$ range, where $\Delta f$ is the band width of the corresponding channel in the radiometer (here $\Delta f=0.3 \mathrm{GHz}$ and the frequency band function has been assumed rectangular, $\left.g_{\omega}=(2 \pi \Delta f)^{-1}\right)$. For comparison, in Fig. 3 both the LFS and the HFS ECE spectra are shown.

Excluding the low frequencies $(f<127 \mathrm{GHz})$, for which the plasma is optically thin, the LFS spectrum does not show any visible difference between the thermal and the non-thermal spectrum. Because of the high optical depth, the "weighted" center of the emission line for each frequency $(127 \mathrm{GHz} \leq f \leq 157 \mathrm{GHz})$ has a very small shift from the "cold" resonance $\left(\omega=2 \omega_{c e}\right)$ position. However, the HFS spectrum has a more pronounced peak at low frequencies, indicating the contribution of the supra-thermal electrons. Despite the fact, that for these frequencies the "cold" resonance position, $\omega=2 \omega_{c e}$, is far from the axis (and may be even outside of the plasma), the "weighted" center of the emission line, being observed from HFS, is located near the axis.

While the cyclotron emissivity is the local characteristic, the energy range of electrons participating in the reabsorption is defined exactly by the direction of observation, and it is quite different for the LFS and HFS cases. In other words, the down-shifted emission of energetic electrons near the axis, which propagates in the HFS direction is not reabsorbed, and can be identified in the measurements. The importance of this difference between the LFS and HFS spectra for the interpretation and for the "mapping" of it onto the radius, is demonstrated in Fig. 4. Here are shown the major radius projections of the weighted center of the emission line, $R_{\text {ece }}$, versus the corresponding "cold" resonance (major radius) position, $R_{\mathrm{cy}}$. As expected for the LFS spectrum, $R_{\text {ece }}$ is very close to $R_{\mathrm{cy}}$, being downshifted by not more than $2 \mathrm{~cm}$. But the HFS spectrum has $R_{\text {ece }}$ strongly shifted below $R_{\text {cy }}$ inside the plasma. In fact, the spatial location of the emission line is dominated by reabsorption, and not by emission.

One can compare also the velocities of electrons, which contribute to the emission (Fig. 5). Due to the strong reabsorption, the bulk electrons with $v / v_{t h} \simeq 1$ are responsible for the LFS ECE spectrum. But for the HFS ECE spectrum, much more energetic electrons contribute to the EC emission, especially in the range $115 \mathrm{GHz} \leq f \leq$ $130 \mathrm{GHz}$, where only (supra-thermal) tails of the distribution function with $v / v_{t h} \sim 3-4$ are responsible. Note, that the difference in electron energies for the LFS and the HFS ECE spectra is quite large for almost all frequencies. One

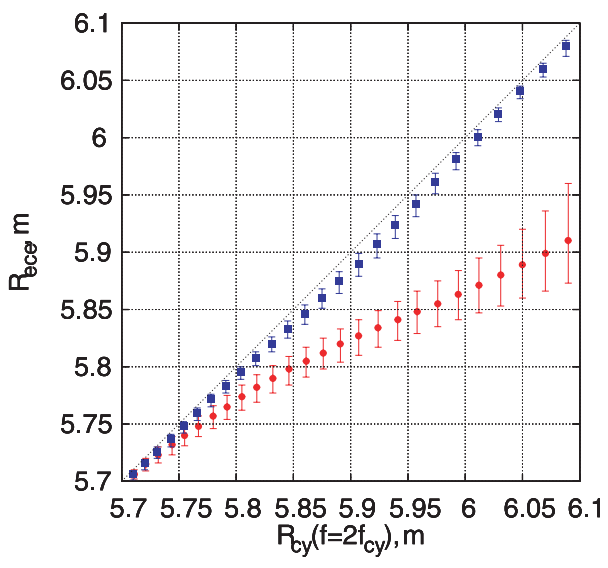

Fig. 4 The center of the emission line $v s$ the "cold" resonance position ( $\left.\omega=2 \omega_{c e}\right)$, projected to the major radius, is shown for both the LFS (blue) and the HFS (red) cases.

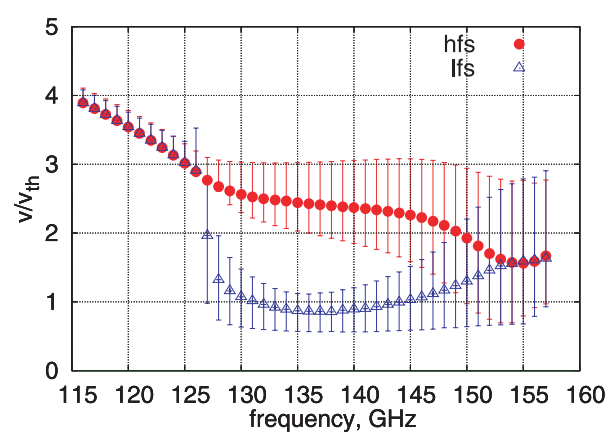

Fig. 5 Velocity ranges (with $\left.v_{t h}=\left(2 T_{e} / m\right)^{1 / 2}\right)$ of emitting electrons as function of frequency. The bars indicate the main velocity range of contributing electrons.

can see also, that the velocity range of the LFS spectra for $f \leq 127 \mathrm{GHz}$ almost coincides with the HFS one, having $v / v_{t h} \sim 3-4$. As was already discussed above, the reason is that the periphery plasma is optically thin for the downshifted 2nd harmonic emission from the (fast) electrons coming in LFS direction from the plasma center.

As expected, the standard LFS observation with X2mode, is the most accurate and convenient method to measure the radiative electron temperature profile. Additional information, related to non-thermal electrons, can be obtained with complementary HFS measurements.

\section{Summary}

TRAVIS is a powerful code which covers a broad area of problems in both heating (ECRH/ECCD) and ECE diagnostic. Thanks to a general model adopted for the Hamiltonian, the kinetic effects which lead to anomalous dispersion are taken into account. Macroscopic quantities such as the deposition profile or the radiative temperature can be decomposed in the contribution from trapped and passing particles. This tool provides a better understanding of kinetics in stellarators, not only in interpreting the exper- 
iments, but also in preparing suitable plasma target and magnetic configuration. The spatial width of the emission line, calculated together with the ECE spectrum, gives the theoretical upper limit to the spatial resolution of ECE diagnostic. The energy range of emitting electrons calculated gives an additional possibility to analyse kinetic effects.

[1] J. Nürenberg and R. Zille, Phys. Letters bf 114A 129 (1986).

[2] V. Erckmann et al., Fusion Sci. Technol., accepted for publication, 51 (2007); Yu. Turkin et al., Fusion Sci. Technol., 50, 387 (2006).

[3] E. Mazzucato, Phys. Fluids B 1, 1855 (1989).

[4] E. Westerhof, Plasma Phys. Control. Fusion 39, 1015 (1997).

[5] M.D. Tokman, E. Westerhof and M.A. Gavrilova, Plasma Phys. Control. Fusion 42, 91 (2000).
[6] M. Bornatici et al., Nucl. Fusion 23, 1153 (1983).

[7] M. Taguchi, Plasma Phys. Control. Fusion 31, 241 (1989).

[8] M. Romé et al., Plasma Phys. Control. Fusion 40, 511 (1998).

[9] Y.R. Lin-Liu, V.S. Chan and R. Prater, Phys. Plasmas 10, 4064 (2003).

[10] M.A. Balakina, M.D. Tokman, O.B. Smoliakova, Plasma Phys. Reports 29, 53 (2003).

[11] N.B. Marushchenko et al., Proc. of the 31st EPS Conference Plasma Phys., London, 28 June - 2 July 2004 ECA Vol.28G P-1.204.

[12] N.B. Marushchenko et al., Fusion Sci. Technol. 50, 395 (2006).

[13] G. Giruzzi, Nucl. Fusion 28, 1413 (1998).

[14] P. Blanchard et al., Plasma Phys. Control. Fusion 44, 2231 (2002).

[15] V. Tribaldos and B.P. van Milligen, Nucl. Fusion 36, 283 (1996). 